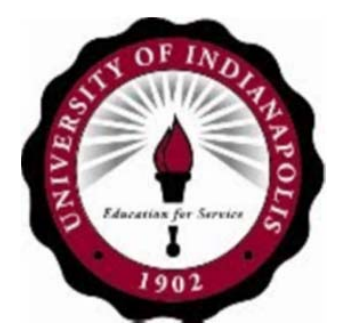

\title{
Unsupportive Social Interactions, Shame, and Psychological Well-Being in People with HIV
}

Amanda R. McErlean, M.A. ${ }^{1}$, Erin M. Fekete, Ph.D. ${ }^{1}$, Stacey L. Williams, Ph.D. ${ }^{2}$,

Matthew D. Skinta, Ph.D. ${ }^{3}$, Nicole M. Taylor, Ph.D. ${ }^{1}$, \& Leah M. Bogusch, B.S. ${ }^{1}$

${ }^{1}$ University of Indianapolis, ${ }^{2}$ East Tennessee State University, ${ }^{3}$ Private Practice, San Francisco, CA

First Author Contact Information: mcerleana@uindy.edu

Principal Investigator Contact Information: feketee@uindy.edu

\section{ABSTRACT}

We hypothesized that unsupportive social interactions (USI) would be associated with poorer psychological wellbeing in people living with HIV (PLWH), and that this relationship would be explained by increased levels of
shame. 106 PLWH completed an online questionnaire including measures of HIV-specific USI, shame depression, negative affect, and perceived stress. Results suggest that insensitive interactions were related to higher levels of depression, negative affect, and perceived stress through higher levels of shame. Internalized feelings of shame may partially explain the relationship between insensitive interactions and psychological well-being.

\section{INTRODUCTION \\ People living with HIV (PLWH) often experience illness- specific unsupportive social interactions (USI), including being blamed for their illness, forcing optimism, being overly critical, and distancing. \\ - USI are associated with poorer physical and psychological well-being \\ HIV-specific USI are a form of felt or enacted stigma, and may be associated with increased feelings of shame about one's illness condition. \\ Shame is an emotion that reflects internal feelings of inferiority, worthlessness, inadequacy, and alienation due to a behavior or a condition. Internalized shame may lead to poorer subjective ratings of overall well-being. \\ Feelings of shame may be a factor that explains the relationship between unsupportive social interactions and poorer well-being. \\ HYPOTHESES \\ HIV-specific USI will be associated with poorer psychological well-being (depression, positive an ave affect, perceived stress) in PLWH. \\ Internalized shame will mediate the relationship between \\ HIV-specific USI and psychological well-being. \\ insensitive interactions will be associated with higher \\ insensilve interactions will be associated with higher \\ poorer psychological well-being}

PARTICIPANTS (N = 106)
\begin{tabular}{|l|l|}
\hline Age (Years) & Mean $=42.6$ \\
\hline Gender & $66 \%$ male, $44 \%$ female \\
\hline Ethnicity & $\begin{array}{l}48.2 \% \text { Black; 35.8\% White; } \\
13.2 \% \text { Hispanic; } 2.8 \% \text { Biracial }\end{array}$ \\
\hline Education & $\begin{array}{l}\text { Did Not Graduate High School }=5.7 \%, \\
\text { High School Graduate }=74.5 \%, \\
\text { College Graduate }=19.8 \%\end{array}$ \\
\hline Yearly Income & Median \$10,000 - \$14,999 \\
\hline $\begin{array}{l}\text { Years since HIV } \\
\text { diagnosis }\end{array}$ & Mean = 11.2 years \\
\hline
\end{tabular}

\begin{tabular}{|c|c|c|c|c|c|}
\hline \multicolumn{6}{|c|}{ PROCEDURE } \\
\hline \multicolumn{6}{|c|}{$\begin{array}{l}\text { Participants completed an online questionnaire and } \\
\text { were compensated with a } \$ 20.00 \text { gift card. } \\
\text { Measures included: } \\
\text { - Demographic Information } \\
\text { - Unsupportive Social Interactions Scale } \\
\text { - Centernalized Shame Scale } \\
\text { Scale (CES-D) } \\
\text { - Negative Affect Subscale of the Positive and } \\
\text { Negative Affect Schedule (PANAS) } \\
\text { - Perceived Stress Scale (PSS) }\end{array}$} \\
\hline \multicolumn{6}{|c|}{ MEASURES } \\
\hline Measure & Mean & SD & $\begin{array}{l}\text { Actual } \\
\text { Range }\end{array}$ & \begin{tabular}{|c|} 
Potential \\
Range
\end{tabular} & $\alpha$ \\
\hline Distancing & 6.57 & 6.2 & $0-24$ & $0-24$ & .91 \\
\hline Blaming & 6.32 & 7.1 & $0-24$ & $0-24$ & .94 \\
\hline $\begin{array}{l}\text { Forced } \\
\text { Optimism }\end{array}$ & 8.63 & 6.7 & $0-24$ & $0-24$ & .89 \\
\hline $\begin{array}{l}\text { Insensitive } \\
\text { Interactions }\end{array}$ & 8.18 & 6.9 & $0-24$ & $0-24$ & .90 \\
\hline $\begin{array}{l}\text { Internalized } \\
\text { Shame }\end{array}$ & 73.29 & 26.5 & $28-139$ & $28-140$ & .96 \\
\hline Depression & 21.45 & 13.6 & $0-54$ & $0-60$ & .92 \\
\hline $\begin{array}{l}\text { Negative } \\
\text { Affect }\end{array}$ & 21.18 & 9.5 & $10-44$ & $10-50$ & .94 \\
\hline $\begin{array}{l}\text { Perceived } \\
\text { Stress }\end{array}$ & 17.95 & 7.9 & $0-38$ & $0-40$ & .81 \\
\hline
\end{tabular}

\section{ANALYSIS PLAN}

- Hierarchical Linear Regression and Mediation Analysis - Predictor Variables: Insensitive Interactions,

Blaming, Forced Optimism, Distancing

- Mediator: Shame

Outcome Variables: Depression, Negative Affect, Perceived Stress

-Covariates

Any sociodemographic, health, or social characteristics associated with the outcome variable

\section{RESULTS}

- Total Effects depression $(\mathrm{b}=.90, \mathrm{SE}=.34, p<.01)$, negative affect $(b=.61, S E=.26, p<.05)$, and perceived stress $(b=.57, S E=.22, p<.05)$.

- No other USI were associated with well-being

Mediation.

Only insensitive interactions operated on depression, negative affect, and perceiv stress through higher levels of shame.

\begin{tabular}{|l|c|c|c|}
\hline $\begin{array}{c}\text { Outcome } \\
\text { Variable }\end{array}$ & $\begin{array}{c}\text { Indirect } \\
\text { Effect }\end{array}$ & $\begin{array}{c}\text { Confidence } \\
\text { Interval }\end{array}$ & $\begin{array}{c}\text { Sobel's z- } \\
\text { Test }\end{array}$ \\
\hline Depression & .73 & .33 to 1.15 & $2.96^{\star \star}$ \\
\hline Negative Affect & .53 & .25 to .84 & $2.93^{\star \star}$ \\
\hline Perceived Stress & .45 & .22 to .71 & $2.93^{\star \star}$ \\
\hline
\end{tabular}

${ }^{p}<<.05 ; * *_{p}<.01 ; * * w_{p}<.001$

DEPRESSION

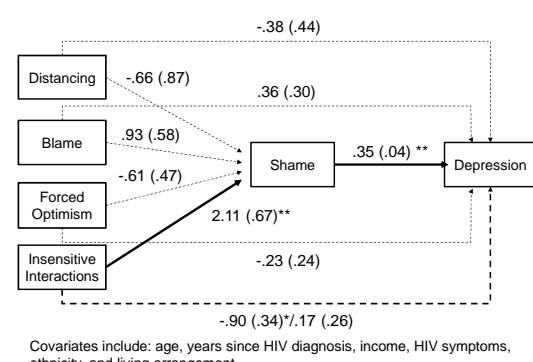

Covariates include: age, years since HIV diagnosis, income, HIV symptoms.
ethnicity, and liven arrangement. $0<0.05 ; *_{p}<.01 ; * w_{p}<.001$
ETSU

East Tennessee State University

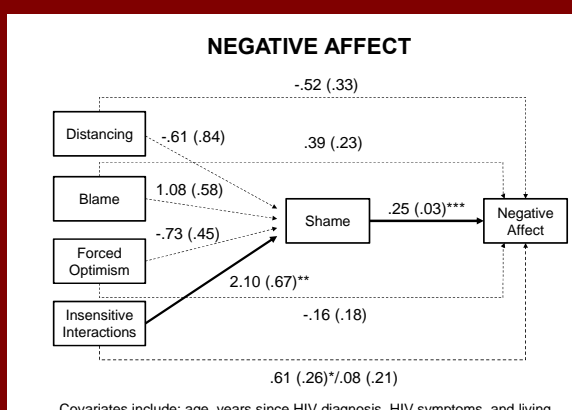

Covariates include: age, years
arrangement.
${ }_{p}<<.05 ; * *_{p}<.01 ; * * x_{p}<.001$

${ }^{*} p<.05 ; * *_{p}<.01 ; * *{ }^{*} p<.001$

PERCEIVED STRESS

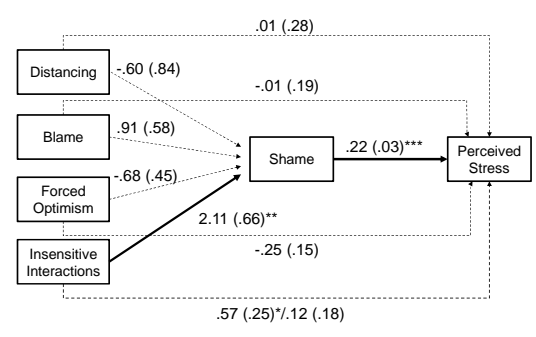

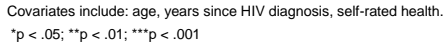

\section{DISCUSSION}

Internalized feelings of shame appear to partially explain the relationship between insensitive interactions and psychological well-being.

Psychotherapeutic interventions targeting internalized shame may have several benefits.

- Reduction in psychological distress.

Lessened effects of insensitive or stigmatizing social interactions on the well-being of

individuals living with HIV.

erience less psychological distress may experience better illness outcomes, be more adherent to medication recommendations, and have slower disease
progression. 